JEEP 2011, 00009 (2011)

DOI: $10.1051 /$ jeep/201100009

(C) Owned by the authors, published by EDP Sciences, 2011

\title{
Conductivité électrique et gap optique des composés ternaires du système Ag-In-Te
}

\author{
Z. Bahari ${ }^{1,2}$, J. Rivet ${ }^{1}$ et J. Dugué ${ }^{1}$ \\ ${ }^{1}$ Laboratoire de chimie physique et minérale, Faculté des sciences pharmaceutiques et biologiques, \\ Université Paris Descartes, 4, avenue de l'Observatoire, 75006 Paris, France \\ ${ }^{2}$ Laboratoire de chimie du solide minéral (LCSM), Faculté des sciences, Université Mohamed $1{ }^{\mathrm{er}}$, \\ Route Sidi Maâfa, B.P. 524, Oujda, Maroc
}

\begin{abstract}
Résumé. Les mesures de conductivité électrique par la méthode de Van Der Paw et du gap optique par spectroscopie IR ont été réalisées sur les composés ternaires $A g I n T e_{2}, \operatorname{AgIn}_{5} \operatorname{Te}_{8}$ et $\mathrm{Ag}_{3} \operatorname{In}_{97} \mathrm{Te}_{147}$. La mesure des constantes de Hall montre que les trois composés ont un comportement semiconducteur de type $\mathrm{p}$.
\end{abstract}

\section{Introduction}

L'étude du diagramme de phase Ag-In-Te [1, 2] a mis en évidence trois composés ternaires, $\operatorname{AgInTe}{ }_{2}, \operatorname{AgIn}_{5} \mathrm{Te}_{8}$ et $\mathrm{Ag}_{3} \mathrm{In}_{97} \mathrm{Te}_{147}$, situés sur la ligne quasi-binaire $\mathrm{Ag}_{2} \mathrm{Te}$ $\mathrm{In}_{2} \mathrm{Te}_{3}[3]$. Après détermination de leur domaine d'existence en température et en composition, et de la structure cristalline du seul composé $\mathrm{AgInTe}_{2}$, nous avons entrepris une étude préliminaire de leurs propriétés électriques.

\section{Protocole expérimental}

Les échantillons sont préparés par fusion des éléments pris en quantité stoechiométrique. Ils subissent ensuite deux recuits pendant une quinzaine de jours, suivis d'une trempe rapide. L'homogénéité des produits obtenus est contrôlée par diffraction des rayons X.

Les mesures de conductivité électrique ont été réalisées par la méthode de Van Der Pauw [4]. La mesure de la constante de Hall nous a permis de déterminer la nature et la densité des porteurs de charge. De l'enregistrement des spectres de transmission entre 800 et $2500 \mathrm{~nm}$ à l'aide d'un spectrophotomètre Cary, nous avons déduit le gap optique de ces composés.

\section{Résultats}

Pour les trois composés $\operatorname{AgInTe}_{2}, \mathrm{AgIn}_{5} \mathrm{Te}_{8}$ et $\mathrm{Ag}_{3} \mathrm{In}_{97} \mathrm{Te}_{147}$, le logarithme de la conductivité varie linéairement en fonction de l'inverse de la température (Figures 1 et 2).
Les échantillons recuits à $600^{\circ} \mathrm{C}$ sont plus résistants que ceux recuits à $400^{\circ} \mathrm{C}$. Les résultats de mesure de la constante de Hall de ces composés dans les deux cas de traitement thermique montrent que la conduction électrique est assurée essentiellement par des trous (semiconducteur de type $\mathrm{p}$ ).

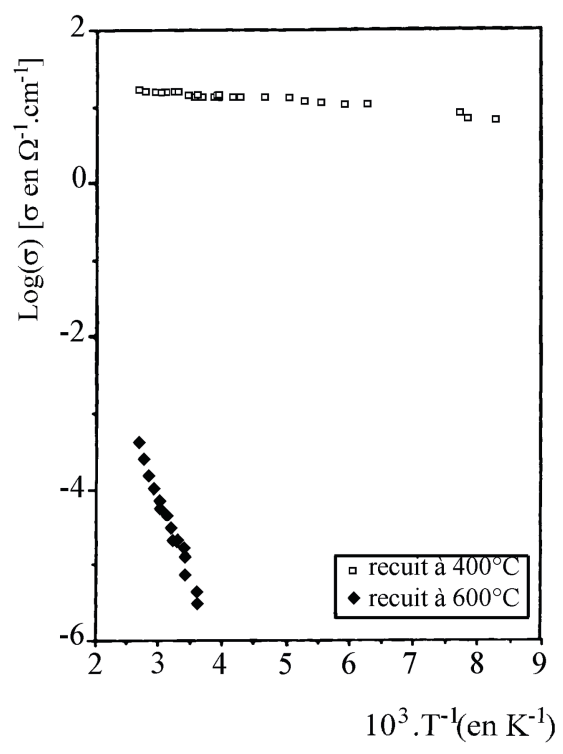

Fig. 1. Variation de la conductivité en fonction de la température pour $\mathrm{AgInTe}_{2}$.

La différence des valeurs de la conductivité s'explique par le nombre des porteurs de charge (Tableau 1). 
Pour vérifier l'effet de la stoechiométrie, nous avons étudié le comportement électrique de quelques échantillons situés à proximité du composé $\operatorname{AgInTe}_{2}$. L'étude a porté essentiellement sur les composés suivants :

- $\mathrm{AgInTe}_{2}$

- $\quad \mathrm{Ag}_{2,133} \operatorname{In}_{2,133} \mathrm{Te}_{5,733}$ (excès de Te)

- $\quad \mathrm{Ag}_{2,4} \mathrm{In}_{2,567} \mathrm{Te}_{5,033}$ (excès de $\mathrm{In}_{2} \mathrm{Te}_{3}$ )

- $\quad \mathrm{Ag}_{2,33} \mathrm{In}_{2,467} \mathrm{Te}_{5,2}$ (excès de Te et de In).

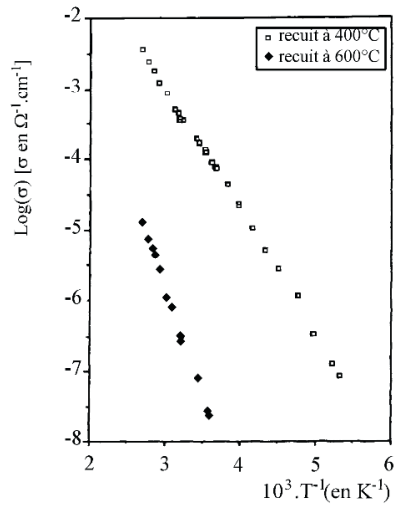

$\operatorname{AgIn}_{5} \operatorname{Te}_{8}$

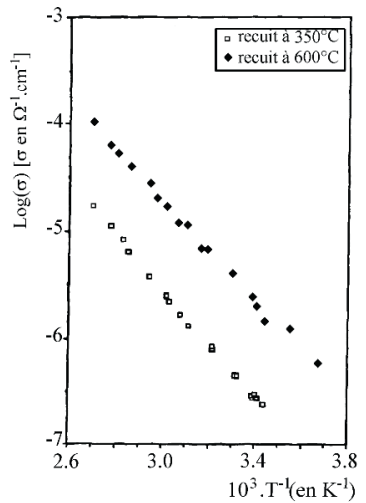

$\mathrm{Ag}_{3} \mathrm{In}_{97} \mathrm{Te}_{147}$
Fig. 2. Variation de la conductivité en fonction de la température pour $A g \operatorname{In}_{5} \mathrm{Te}_{8}$ et $\mathrm{Ag}_{3} \operatorname{In}_{97} \mathrm{Te}_{147}$.

Tableau 1. Conductivité électrique et densité des porteurs de charge mesurées à $295 \mathrm{~K}$.

\begin{tabular}{|c|c|c|c|}
\hline Composé & Recuit $\left({ }^{\circ} \mathrm{C}\right)$ & $\sigma\left(\Omega^{-1} \cdot \mathrm{cm}^{-1}\right)$ & $\mathrm{p}\left(\mathrm{cm}^{-3}\right)$ \\
\hline \multirow{2}{*}{$\mathrm{AgInTe}_{2}$} & 600 & $5,5 \cdot 10^{-6}$ & $1,7.10^{11}$ \\
\hline & 400 & 11,6 & $2,6.10^{17}$ \\
\hline \multirow{2}{*}{$\mathrm{AgIn}_{5} \mathrm{Te}_{8}$} & 600 & $3,1.10^{-5}$ & $2,0.10^{12}$ \\
\hline & 400 & $2,0.10^{-4}$ & $8,6.10^{12}$ \\
\hline $\mathrm{Ag}_{3} \mathrm{In}_{97} \mathrm{Te}_{147}$ & 350 & $6,5 \cdot 10^{-7}$ & $5,0.10^{10}$ \\
\hline
\end{tabular}

La figure 3 montre que l'addition de tellure ou d'indium augmente la conductivité électrique. Ceci peut s'expliquer par l'augmentation du taux de lacunes cationiques ou par l'augmentation de la teneur en indium sur le site de l'argent (substitution cationique). Dans ce dernier cas, on peut imaginer par jeu de transfert de charges $\mathrm{Ag}^{+} \rightarrow \mathrm{In}^{3+}+2 \mathrm{e}^{-}$que la conductivité deviendra de type n. En effet, la mesure de la constante de Hall à température ambiante sur l'échantillon avec excès de Te et de In confirme le caractère d'un semi conducteur de type $n$.

Des mesures de gap optiques ont été réalisées sur les deux composés $A g I n T e e_{2}$ et $\mathrm{Ag}_{3} \mathrm{In}_{97} \mathrm{Te}_{147}$. La figure 4 représente les spectres de transmission pour ces deux composés entre 1000 et $2500 \mathrm{~nm}$ qui mettent en évidence les transitions interbandes directes. La relation entre le coefficient d'absorption $\alpha$ et $\left(h v-E_{g}\right)$ est de la forme : $\alpha=\alpha_{0}\left(h v-E_{g}\right)^{\frac{1}{2}}$.

Le seuil d'absorption $v_{\text {seuil }}$ détermine la largeur optique de la bande interdite par la relation $E_{g}=h v_{\text {seuil }}$. Le calcul de

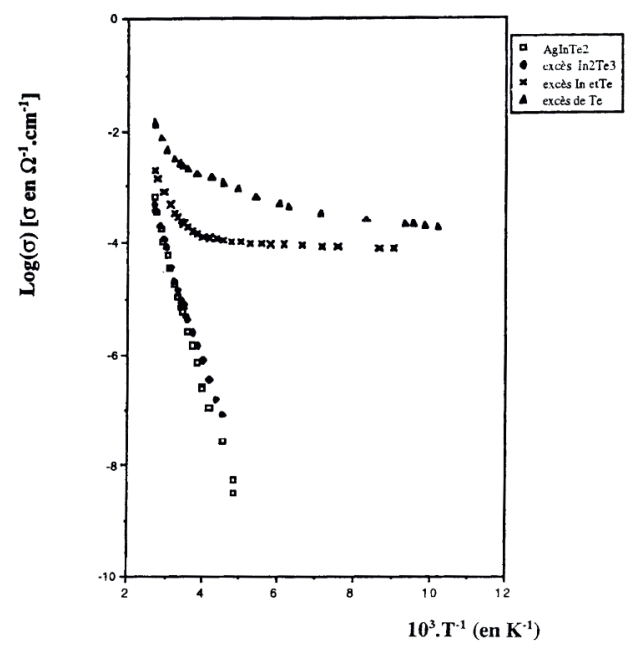

Fig. 3. Variation de la conductivité en fonction de la température d'échantillons voisins de $\mathrm{AgInTe}_{2}$.
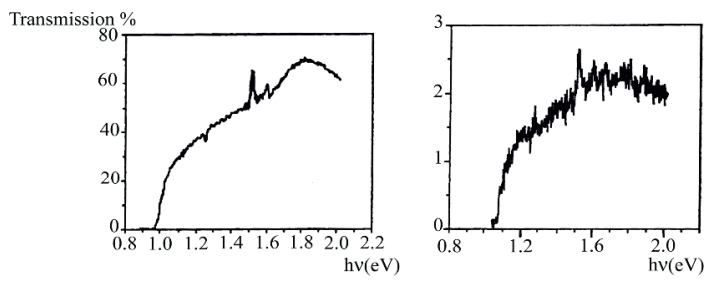

$\mathrm{Ag}_{3} \mathrm{In}_{97} \mathrm{Te}_{147}$

$\mathrm{AgInTe}_{2}$

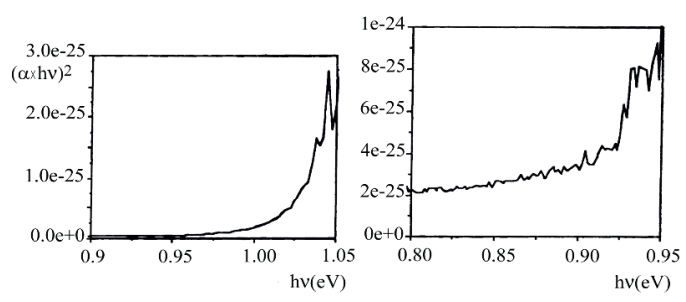

Fig. 4 Spectres de transmission des composés $\mathrm{Ag}_{3} \mathrm{In}_{97} \mathrm{Te}_{147}$ et $\mathrm{AgInTe}_{2}$ (en haut) et tracé suivant la formule $(\alpha \times h v)^{2}$ en fonction de hv (en bas).

la largeur de cette bande interdite donne une valeur comparable à celle que l'on obtient par les mesures électriques pour les deux composés étudiés (Tab. 2).

Tableau 2. Gaps optique et électrique (eV).

\begin{tabular}{|l|c|c|}
\hline \multicolumn{1}{|c|}{ Composé } & Gap optique & Gap électrique \\
\hline $\mathrm{AgInTe}_{2}$ & 0,92 & 0,93 \\
\hline $\mathrm{Ag}_{3} \mathrm{In}_{97} \mathrm{Te}_{147}$ & 1,02 & 0,99 \\
\hline
\end{tabular}


On remarque que le composé $\mathrm{Ag}_{3} \mathrm{In}_{97} \mathrm{Te}_{147}$ transmet beaucoup plus que le composé $\mathrm{AgInTe}_{2}$. Ceci tient à la qualité de la surface du matériau : la présence de fissures au niveau de l'échantillon réfléchit le rayonnement et par suite le matériau ne transmet pas.

\section{Conclusion}

Ces composés manifestent un caractère semi-conducteur intrinsèque. Cependant, comme on sait qu'un tel caractère ne peut exister dans le cas de notre étude, nous estimons qu'il pourrait peut-être s'agir d'un caractère semi-conducteur extrinsèque avec compensation des défauts.

\section{Références}

1. Z. Bahari, J. Rivet, B. Legendre et J. Dugué, J. Alloys Compounds, 282 (1999), 164-174

2. Z. Bahari, J. Rivet, B. Legendre et J. Dugué, J. Alloys Compounds, 289(1999), 99-115

3. Z. Bahari, J. Rivet et J. Dugué, C. R. Acad. Sci. Paris, série II C, 411-415, (1998) 